\title{
Searches for sterile neutrinos with IceCube DeepCore
}

\author{
Soebur Razzaque ${ }^{1,2, \text { f }}$ and A. Yu. Smirnov ${ }^{3, \dagger}$ \\ ${ }^{1}$ School of Physics, Astronomy and Computational Sciences, \\ George Mason University, Fairfax, VA 22030, USA \\ ${ }^{2}$ Resident at the Space Science Division, US Naval Research Laboratory, Washington, DC 20375, USA \\ ${ }^{3}$ The Abdus Salam International Centre for Theoretical Physics, I-34100 Trieste, Italy
}

(Dated: October 29, 2018)

\begin{abstract}
We show that study of the atmospheric neutrinos in the 10-100 GeV energy range by DeepCore sub-array of the IceCube Neutrino Observatory can substantially constrain the mixing of sterile neutrinos of mass $\sim 1 \mathrm{eV}$ with active neutrinos. In the scheme with one sterile neutrino we calculate $\nu_{\mu}-$ and $\bar{\nu}_{\mu}-$ oscillation probabilities as well as zenith angle distributions of $\nu_{\mu}^{C C}$ (charge current) events in different energy intervals in DeepCore. The distributions depend on the mass hierarchy of active neutrinos. Therefore, in principle, the hierarchy can be identified, if $\nu_{s}$ exists. After a few years of exposure the DeepCore data will allow to exclude the mixing $\left|U_{\mu 4}\right|^{2} \geq 0.02$ indicated by the LSND/MiniBooNE results. Combination of the DeepCore and high energy IceCube data will further improve sensitivity to $\nu_{s}$ mixing parameters.
\end{abstract}

PACS numbers: 14.60.Pq, 14.60.St

\section{INTRODUCTION}

Mixing of sterile neutrinos of $\mathrm{eV}$ mass scale with active neutrinos, as is indicated by the LSND/MiniBooNE results [1, 2], significantly affects the atmospheric neutrino fluxes 3 7]. The primary effect arises in the TeV energy range due to the MSW resonant enhancement of $\bar{\nu}_{\mu}-\bar{\nu}_{s}$ oscillations while neutrinos pass through the matter of the Earth. As a result, the $\bar{\nu}_{\mu}$-flux, and consequently, number of $\nu_{\mu}$ - events at the detectors deplete. The oscillations lead to distortion of the zenith angle and energy distributions of events.

In this paper we will show that the atmospheric neutrino fluxes at low energies, $E<0.5 \mathrm{TeV}$, are also strongly affected by the $\nu_{s}$ oscillations in spite of the fact that the resonance enhancement occurs in the $\mathrm{TeV}$ range. Therefore studies of the low energy fluxes can substantially contribute to searches for sterile neutrinos. With a densely spaced array of phototubes in the middle of the IceCube array, DeepCore sub-array [8, 9] significantly enhances IceCube sensitivity to $(10-100) \mathrm{GeV}$ neutrinos. This makes possible to study the atmospheric neutrino oscillations with DeepCore at low energies 10 13.

As it was realized in Ref. [5], the $\nu_{s}$ effects depend not only on $U_{\mu 4}$ relevant for the LSND/MiniBooNE results but also on the $\nu_{\tau}$ admixture $U_{\tau 4}$. There are two special cases: (i) $U_{\tau 4}=0$, which corresponds to the so called flavor-mixing scheme, and (ii) $U_{\tau 4}=U_{\mu 4}$ which determines the mass-mixing scheme for maximal 2-3 mixing. In the latter case $\nu_{s}$ mixes with the active neutrinos through the mass eigenstate $\nu_{3}$. At energies below $0.5 \mathrm{TeV}$ in the $\nu_{s}$ mass-mixing scheme the $\nu_{s}$ effects are

\footnotetext{
*Electronic address: srazzaqu@gmu.edu
}

† Electronic address: smirnov@ictp.it strong, whereas in the $\nu_{s}$ flavor-mixing scheme the oscillation probabilities are unaffected [5]. In this paper for illustration we consider a scheme with one sterile neutrino, as was favored by recent MINOS data 14, 15, and global fit [16] and focus on the $\nu_{s}$ mass-mixing scheme. We show that the mixing angle with the active neutrinos can be significantly constrained by $(10-100) \mathrm{GeV}$ atmospheric neutrino data collected in DeepCore [8].

We present oscillation probabilities in the $\nu_{s}$ massmixing scheme at low energies in Sec. II. In Sec. III we calculate the $\nu_{\mu}^{C C}$ event rate in DeepCore produced by atmospheric neutrinos. We study the zenith and energy distributions of these events. We estimate the sensitivity of DeepCore to the $\nu_{s}$ mixing with active neutrinos in Sec. IV. Sec. V contains discussion and conclusions.

\section{OSCILLATION PROBABILITIES}

At high energies to a good approximation one can consider mixing of the three flavor states $\nu_{f}^{T} \equiv\left(\nu_{s}, \nu_{\tau}, \nu_{\mu}\right)$ in the mass eigenstates $\nu_{\text {mass }}^{T} \equiv\left(\nu_{4}, \nu_{3}, \nu_{2}\right)$, neglecting the mixing of $\nu_{1}\left[5\right.$. In the $\nu_{s}$ mass-mixing scheme, the mixing matrix $U_{f}$, defined as $\nu_{f}=U_{f} \nu_{\text {mass }}$, depends on the angle $\theta_{23}$ and new "sterile" angle $\alpha$, so that in $\nu_{4}$

$$
U_{s 4}=\cos \alpha, U_{\mu 4}=\sin \alpha \sin \theta_{23}, U_{\tau 4}=-\sin \alpha \cos \theta_{23} .
$$

For $\alpha=0$, when the state $\nu_{4}$ decouples and the $3 \nu$-evolution reduces to $2 \nu$-evolution, the $\nu_{\mu}$ survival probability is given by

$$
P_{\mu \mu}=1-\sin ^{2} 2 \theta_{23} \sin ^{2} \frac{\Delta m_{32}^{2} x}{4 E} .
$$

Here $x=2 R_{\oplus}\left|\cos \theta_{z}\right|$ is the length of neutrino trajectory characterized by the zenith angle $\theta_{z}$, and $R_{\oplus}$ is the radius of the Earth. In our numerical estimations we use $\Delta m_{32}^{2}=(2.3-2.5) \cdot 10^{-3} \mathrm{eV}^{2}$ and $\sin ^{2} 2 \theta_{23}=1$. 
According to Eq. (1) the first oscillation minimum (dip) is at energy

$$
E_{\mathrm{min}}^{0}=\frac{1}{\pi} \Delta m_{32}^{2} R_{\oplus} \cos \theta_{z}
$$

For neutrinos moving along the Earth's diameter, $\theta_{z}=\pi$, we obtain $E_{\min }^{0} \approx 25.7 \mathrm{GeV}$.

If $\alpha \neq 0$, the oscillation probability changes substantially due to matter effect. For $E>15 \mathrm{GeV}$ to a good approximation the effect is described by oscillations in uniform medium with the average density along the neutrino trajectory. In turn, for constant density and for energies below $0.5 \mathrm{TeV}$ the probability is given by 5 ]

$$
\begin{aligned}
P_{\mu \mu}= & 1-\cos ^{2} \alpha \sin ^{2} 2 \theta_{23} \\
& \times \sin ^{2}\left[\left(\frac{\Delta m_{32}^{2}}{2 E} \pm\left|V_{\mu}\left(\theta_{z}\right)\right| \sin ^{2} \alpha\right) R_{\oplus} \cos \theta_{z}\right] \\
& -0.5 \sin ^{2} 2 \alpha \sin ^{4} \theta_{23}-0.5 \sin ^{2} \alpha \sin ^{2} 2 \theta_{23}
\end{aligned}
$$

after averaging over fast oscillations driven by the mass squared difference $\Delta m_{43}^{2} \sim 1 \mathrm{eV}^{2}$. Here $V_{\mu}\left(\theta_{z}\right)$ is the average matter potential: $V_{\mu}\left(\theta_{z}\right)=-G_{F} n_{n}\left(\theta_{z}\right) / \sqrt{2}$, and $n_{n}\left(\theta_{z}\right)$ is the average number density of neutrons along the $\theta_{z}$ trajectory. The lower "-" (the upper "+") sign in front of $\left|V_{\mu}\right|$ in Eq. (3) corresponds to antineutrinos (neutrinos). Thus, in the first approximation the $\nu_{s}$ effect is reduced to appearance of an additional contribution to the oscillation phase. This contribution has different signs in the $\nu$ - and $\bar{\nu}$-channels as well as for normal $(\mathrm{NH})$ and inverted $(\mathrm{IH})$ mass hierarchies of the active neutrinos (i.e. for the positive and negative signs of $\left.\Delta m_{32}^{2}\right)$.

According to Eq. (3) the energy of the first oscillation dip changes due to $\nu_{s}$ mixing as

$$
\begin{aligned}
E_{\min } & \approx \frac{\left|\Delta m_{32}^{2}\right|}{\frac{\pi}{R_{\oplus} \cos \theta_{z}} \mp 2\left|V_{\mu}\left(\cos \theta_{z}\right)\right| \sin ^{2} \alpha} \\
& =\frac{E_{\min }^{0}}{1 \mp \frac{2}{\pi}\left|V_{\mu}\left(\cos \theta_{z}\right)\right| R_{\oplus} \sin ^{2} \alpha \cos \theta_{z}},
\end{aligned}
$$

where the lower sign in denominator corresponds to antineutrinos for the normal mass hierarchy and to neutrinos for the inverted mass hierarchy. For $\sin ^{2} \alpha=0.04$, fixed $\Delta m_{32}^{2}, \mathrm{NH}$ and $\cos \theta_{z}=-1$ (an average density $\sim 8.4 \mathrm{~g} \mathrm{~cm}^{-3}$ ) the oscillation dips are at $29.0 \mathrm{GeV}$ and $22.4 \mathrm{GeV}$ for neutrinos and antineutrinos, respectively. This $\nu_{s}$ induced shift of the $\nu_{\mu}-\nu_{\mu}$ oscillation dip can be used to constrain the mixing angle $\alpha$.

We computed the oscillation probabilities as functions of neutrino energy and zenith angle by numerically solving the $3 \nu$-evolution equation [5] with the density profile given by the Preliminary Reference Earth Model [18]. The features of the obtained results can be easily understood with Eqs. (1) to (4).

Figure 1 shows the $\nu_{\mu} \rightarrow \nu_{\mu}$ (top panel) and $\nu_{\mu} \rightarrow \nu_{\tau}$ (bottom panel) oscillation probabilities as functions of neutrino energy for different $\Delta m_{43}^{2}$ but fixed zenith angle

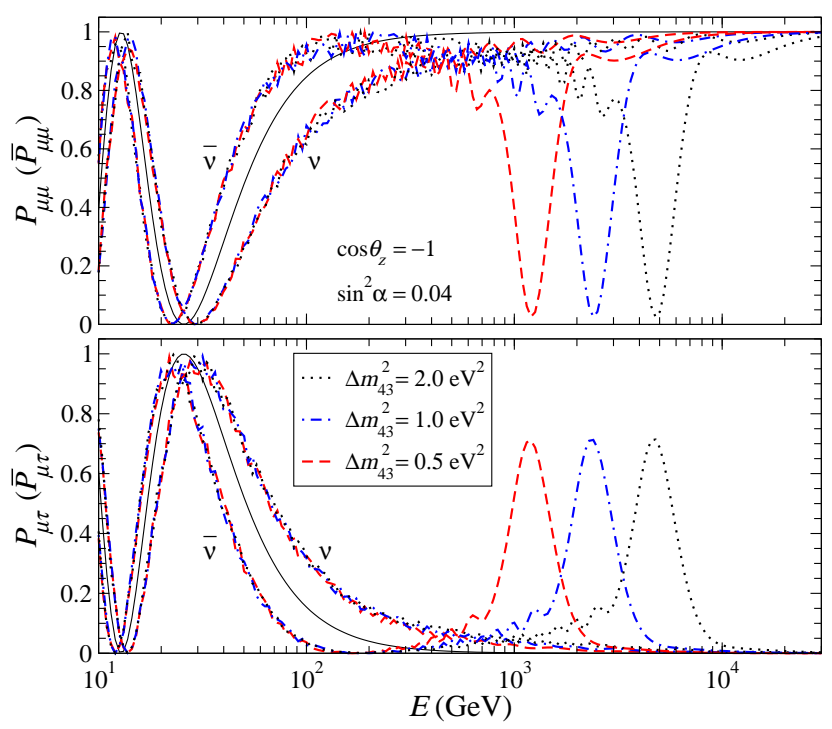

FIG. 1: Oscillation probabilities $\nu_{\mu} \rightarrow \nu_{\mu}$ (top panel) and $\nu_{\mu} \rightarrow \nu_{\tau}$ (bottom panel) for different values of $\Delta m_{43}^{2}$. The solid (broken) lines correspond to the probabilities without (with) $\nu_{s}$ mixing. The normal mass hierarchy is assumed.
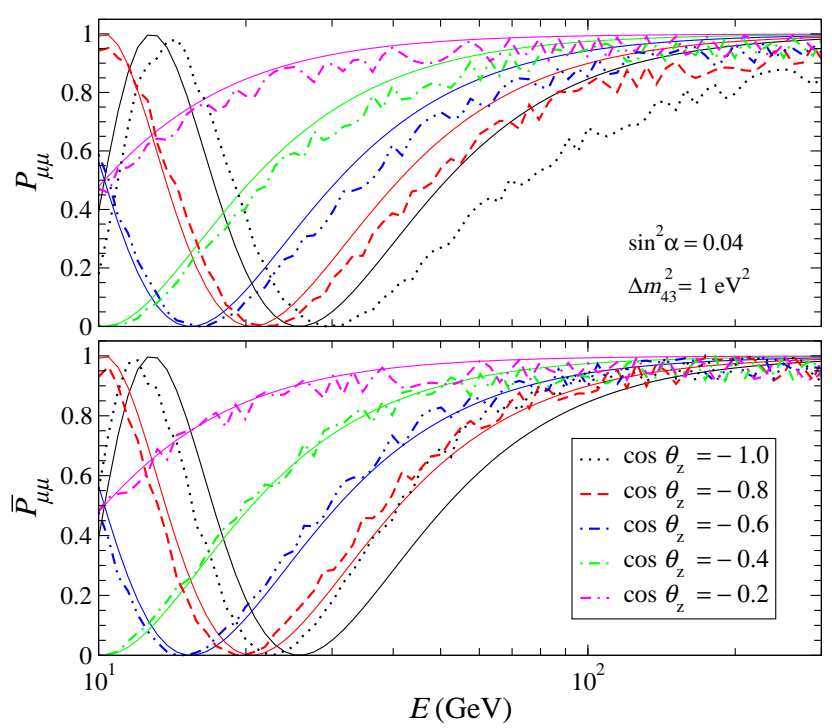

FIG. 2: The survival probabilities of $\nu_{\mu}$ (top panel) and $\bar{\nu}_{\mu}$ (bottom panel) for different values of the zenith angle. The solid (broken) lines correspond to the probabilities without (with) $\nu_{s}$ mixing. The normal mass hierarchy is assumed.

$\theta_{z}=\pi$. The energies of the oscillation dips are in good agreement with the approximate values from Eqs. (2) and (4). According to Fig. 1 the energy of resonant oscillation dip in the $\bar{\nu}-$ channel is proportional to $\Delta m_{43}^{2}$. In contrast, at energies $\lesssim 100 \mathrm{GeV}$ the probabilities depend on $\Delta m_{43}^{2}$ very weakly, which can be seen explicitly in Eq. (3). From the bottom panels, we find that at low energies $\nu_{\mu}$ mainly transforms to $\nu_{\tau}$, whereas at high energies $\nu_{\mu} \rightarrow \nu_{s}$ transition is significant. 

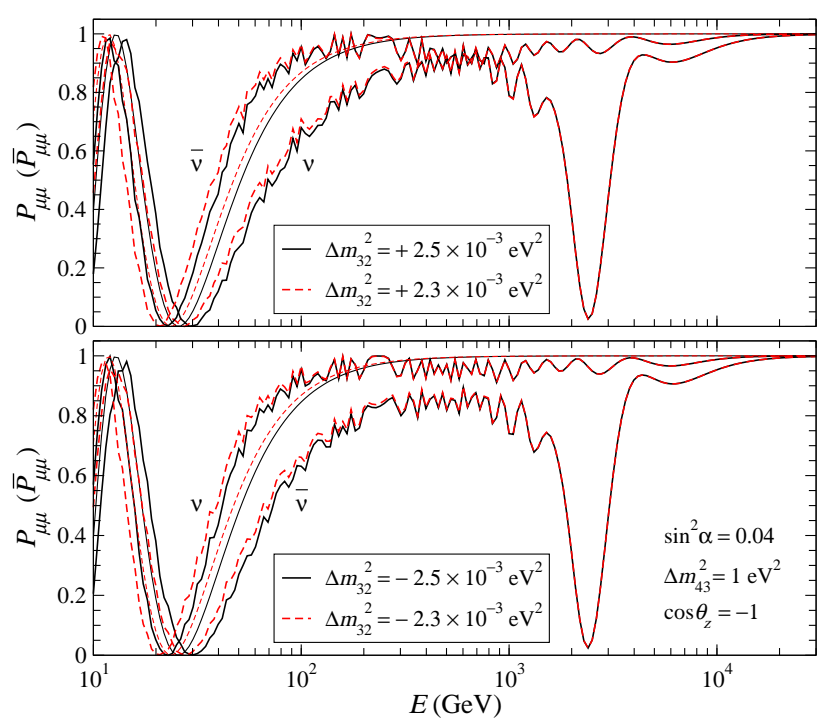

FIG. 3: The $\nu_{\mu}-$ and $\bar{\nu}_{\mu}-$ survival probabilities for two different absolute values of $\Delta m_{32}^{2}$. Top panel: normal mass hierarchy, $\Delta m_{32}^{2}>0$; bottom panel: inverted mass hierarchy, $\Delta m_{32}^{2}<0$. Thin lines are for probabilities without $\nu_{s}$ mixing.

Figure 2 shows the probabilities $P_{\mu \mu}$ and $\bar{P}_{\mu \mu}$ as functions of neutrino energy for different values of $\cos \theta_{z}$, but fixed $\sin ^{2} \alpha$ and $\Delta m_{43}^{2}$ and for NH. With decrease of $\left|\cos \theta_{z}\right|$ the length of the neutrino trajectory decreases and $E_{\min }$ becomes smaller. Furthermore, the difference of probabilities with and without $\nu_{s}$ decreases.

In Figure 3 we show dependence of the survival probabilities on the absolute value of $\Delta m_{32}^{2}$ and on its sign, i.e. on mass hierarchy of active neutrinos. A variation of $\Delta m_{32}^{2}$ also shifts the dip. However, due to dependence of $V$ on $\theta_{z}$ and energy dependence of the usual $\left(\propto \Delta m_{32}^{2}\right)$ term in Eq. (3) the effects of $\Delta m_{32}^{2}$ variations and of sterile neutrino are different. Thus, studies of the energy and zenith angle dependence of the $\nu_{\mu}$ event rates will allow to disentangle the two effects. Change of the mass hierarchy leads to interchange of $\nu$ and $\bar{\nu}$ probabilities at low energies. As a result, for $\mathrm{IH}$ the $\bar{\nu}$ probability is below the $\nu$ probability in whole energy range, and there is no intersection of the two at $0.5-0.7 \mathrm{TeV}$. As follows from Figure 3, the effect of $\Delta m_{32}^{2}$ uncertainty (within $68 \%$ CL region allowed by the MINOS results [17]) on the probabilities, is relatively small. However, it becomes significant when events from $\nu$ and $\bar{\nu}$ interactions sum up.

\section{EVENT RATES IN DEEPCORE}

We calculate the $\nu_{\mu}^{C C}-$ (charged current) event rate in DeepCore. The $\nu_{\mu}$-flux at the detector equals $\Phi_{\mu}=$ $\Phi_{\mu}^{0} P_{\mu \mu}+\Phi_{e}^{0} P_{e \mu} \approx \Phi_{\mu}^{0} P_{\mu \mu}$, where the original atmospheric $\nu_{\mu}$-flux, $\Phi_{\mu}^{0}$, is larger than the original $\nu_{e}-$ flux, $\Phi_{e}^{0}$, by a factor $>10$ in the energy range under consideration. Furthermore, $P_{e \mu} \ll 1$ and $\nu_{e}$ mostly converts into $\nu_{s}[5$.
So, we will neglect the effect of $\nu_{e} \rightarrow \nu_{\mu}$ transition. However, we do take into account contributions from the tau leptons, created by the $\nu_{\tau} N$ interactions. Tau leptons decay into muons with branching ratio $\epsilon \sim 0.18$ being recorded as $\nu_{\mu}^{C C}$-events. The $\nu_{\tau}$-flux at the detector equals $\Phi_{\tau}=\Phi_{\mu}^{0} P_{\mu \tau}$. To be detected as a $\nu_{\mu}^{C C}$ - event in the same muon energy bin the $\nu_{\tau}$ energy needs to be $\eta \sim 2.5$ times higher than a $\nu_{\mu}$ energy. Thus, the rate of $\nu_{\mu}^{C C}-$ events in the neutrino energy and zenith angle bin $i j$ equals

$$
\begin{aligned}
N_{i, j} & =2 \pi \int_{\Delta_{i} \cos \theta_{z}} d \cos \theta_{z} \int_{\Delta_{j} E} d E A_{\mathrm{eff}}\left(E, \theta_{z}\right) \\
& \times\left[\Phi_{\mu}^{0}\left(E, \theta_{z}\right) P_{\mu \mu}\left(E, \theta_{z}\right)+\Phi_{\mu}^{0}\left(\eta E, \theta_{z}\right) \epsilon P_{\mu \tau}\left(\eta E, \theta_{z}\right)\right] \\
& + \text { antineutrinos. }
\end{aligned}
$$

We use the DeepCore "Filter" effective area $A_{\text {eff }}$ from Ref. [8] and the atmospheric neutrino fluxes model of Ref. [19]. The DeepCore effective area is largely insensitive to the zenith angle $\left[20\right.$ so that $A_{\text {eff }}\left(E, \theta_{z}\right) \approx A_{\text {eff }}(E)$. The contribution of the $\tau$-decays to the total $\nu_{\mu}^{C C}$-event rate (the second term in Eq. (5p) is $\lesssim 5 \%$. Since at low energies secondary leptons from the $\nu N$ interactions are scattered at larger angle from the directions of the primary neutrinos, we use large zenith angle bins $\Delta_{i}\left(\cos \theta_{z}\right)=0.2$, as compared to the bins for $\gtrsim 100 \mathrm{GeV}$ data 21]. We assume that for $\nu_{\mu}^{C C}$ events the neutrino energy will be reconstructed with factor of 2 accuracy which implies detection of both muon and the associated (hadron) cascade. Therefore we use the energy bins $\Delta_{j} E=(E \div 2 E)$.

Figure 4 shows the zenith angle distributions of $\nu_{\mu}^{C C}$-events at DeepCore in different energy intervals. The histograms represent the event rates with $\nu_{s}$ and without $\nu_{s}$ mixing correspondingly for $\mathrm{NH}$ (left) and $\mathrm{IH}$ (right). The rates decrease with increase of $\left|\cos \theta_{z}\right|$ which reflects the dip in the oscillation probability. The decrease is steeper in the low energy range which covers the minimum of the dip. With decrease of the $\nu_{s}-$ mixing the difference of histograms decreases as $\sin ^{2} \alpha$. Due to summation of the neutrino and antineutrino signals the total $\nu_{s}$-effect on the number of events is smaller than the effect on probabilities. Still the compensation is not complete due to difference of the original fluxes and crosssections of the neutrinos and antineutrinos. For IH the effect is smaller than for $\mathrm{NH}$, especially for deep (near vertical) trajectories. The reason is that for IH relative deviation of the $\nu$ survival probability from that without $\nu_{s}$-mixing is smaller than deviation for $\bar{\nu}$ probability (see Fig. 3). This is compensated by larger flux and cross section of $\nu$. Notice that difference of the event numbers in each of the zenith angle bins can reach $2-3 \sigma$ after 1 year exposure.

We define the suppression factor as the ratio of the rate $N_{i, j}$ with $\nu_{s}$-mixing to the same rate without $\nu_{s}$ mixing (see also Ref. [5]). Figures 5 and 6 show the suppression factors as functions of zenith angle in different $\nu_{\mu}$ energy ranges for $\mathrm{NH}$ and $\mathrm{IH}$ correspondingly. As can 


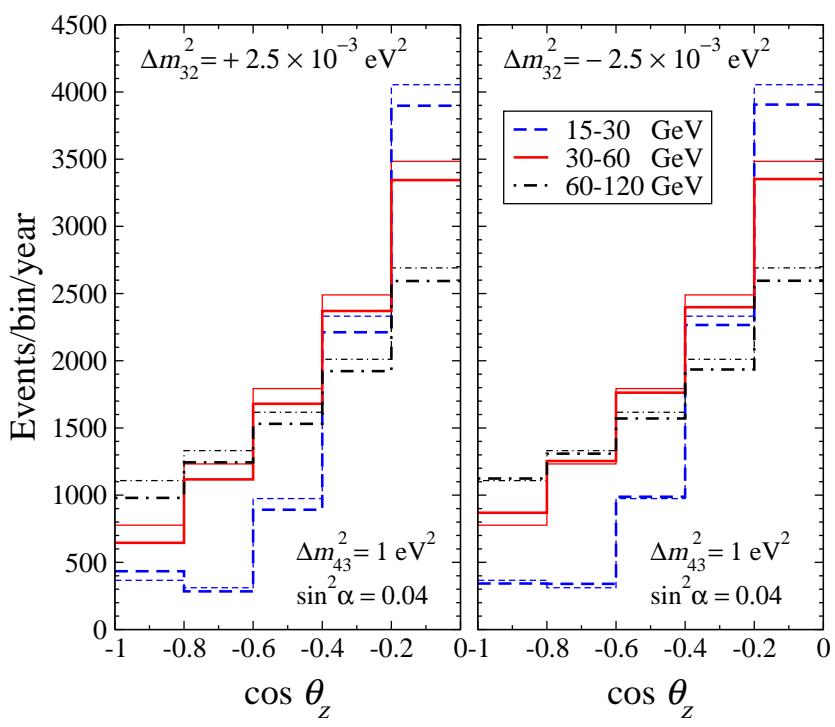

FIG. 4: The $\nu_{\mu}^{C C}$ event rates in different energy and zenith angle bins at IceCube DeepCore. The histograms with thick lines correspond to events with $\nu_{s}$-mixing while the histograms with thin lines correspond to events without $\nu_{s}-$ mixing. Left panel: normal mass hierarchy, right panel: inverted mass hierarchy.

be seen from Fig. 5 for $\mathrm{NH}$ the $\nu_{s}$ mixing mostly affects the vertically upcoming events in the lowest, 15-30 $\mathrm{GeV}$, energy bin. The excess of events in this bin is due to enhanced $\bar{\nu}_{\mu}$-survival probability $\bar{P}_{\mu \mu}$ near the oscillation minimum at $\sim 30 \mathrm{GeV}$ (see Figs. 1 and 2 ). For $\mathrm{IH}$ (Fig. 6) the next bin $(-0.8 \div-0.6)$ rate is equally affected. The distributions depend on $\Delta m_{32}^{2}$ weakly.

In the bottom panels of Figures 5 and 6 we show possible modification of the ratio due to inprecise knowledge of $\Delta m_{32}^{2}$. This is illustrated by the ratio of the two distributions without $\nu_{s}$ for two different values of $\Delta m_{32}^{2}$ : the "true" (observed) value and the "fit" value. Variations of $\Delta m_{32}^{2}$ produce shift of the dip and therefore qualitatively similar distortion of the ratio. Quantitatively the effects are different: the effect of $\Delta m_{32}^{2}$ variations is smaller than the one of $\nu_{s}$ at high energies. For IH and $\left|\cos \theta_{z}\right|>0.4$ the two effects have opposite signs, etc.. In analysis of data one should use $\Delta m_{32}^{2}$ as fit parameter varying in the range allowed by accelerator experiment measurements. In future $\Delta m_{32}^{2}$ will be determined with accuracy $10^{-4}$ $\mathrm{eV}^{2}$ which will substantially reduce the uncertainty.

\section{ESTIMATION OF SENSITIVITY TO MIXING}

To estimate sensitivity of the DeepCore to sterile neutrinos we perform a simple $\chi^{2}$ analysis of the data generated in assumption of zero $\nu_{s}$ mixing. We assume that $\Delta m_{32}^{2}$ is known precisely. We fit these "data" with number of events obtained in the presence of $\nu_{s}$-mixing. To

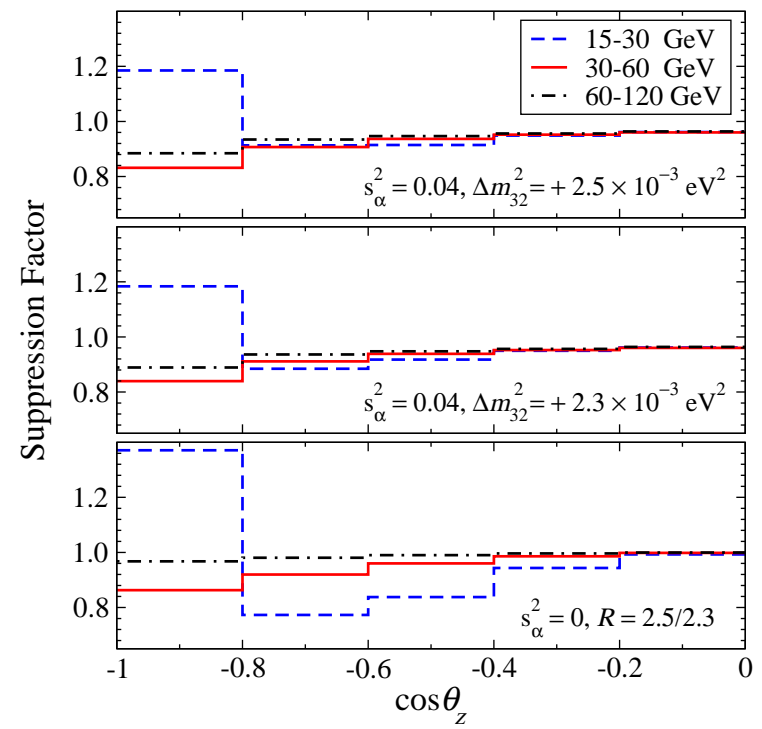

FIG. 5: The suppression factor of $\nu_{\mu}^{C C}$-events as function of the zenith angle for different energy intervals and two values of $\Delta m_{32}^{2}$ (top and middle panels). The bottom panel shows the ratio of event rates for two different $\Delta m_{32}^{2}$ without sterile neutrinos. The normal mass hierarchy is assumed.

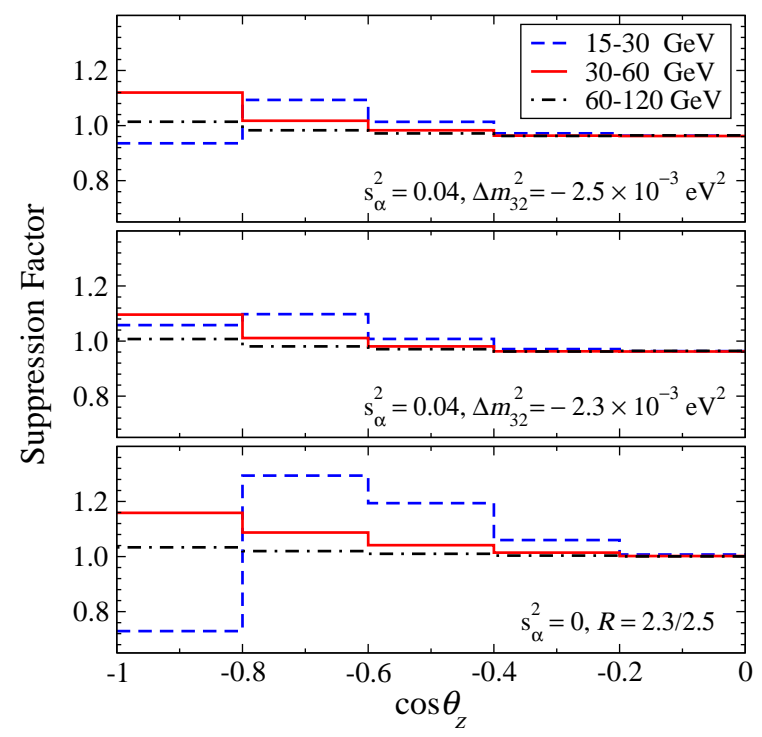

FIG. 6: The same as in Fig. 5 for the inverted mass hierarchy.

take into account possible systematic errors, as in Ref. [5], we introduce an overall normalization factor $C$ and a tilt parameter $\tau$ for the zenith angle distribution of the event rates in Eq. (5) as

$$
N_{i, j}^{\bmod }\left(C, \tau ; \sin ^{2} \alpha\right)=C\left[1+\tau\left(\cos \theta_{i}+0.5\right)\right] N_{i, j}\left(\sin ^{2} \alpha\right) .
$$

We use $\Delta m_{43}^{2}=1 \mathrm{eV}^{2}$. Defining the "null" distribution of zenith angle events without $\nu_{s}-$ mixing as $N_{i, j}^{\text {null }} \equiv$ 
TABLE I: Minimal value $\chi^{2}$ and the corresponding values of the normalization $C$ and tilt parameter $\tau$ from the analysis of the tentative energy and zenith angle distributions of DeepCore event rates with and without $\nu_{s}$ mixing. The values within brackets are calculated using $5 \%$ uncorrelated systematic uncertainties in addition to the statistical and overall normalization uncertainties. The analysis is performed for two different values of mixing angle $\alpha$ and two exposure times.

\begin{tabular}{ccccc}
\hline \hline $\sin ^{2} \alpha$ & Exposure & $\chi_{\min }^{2}$ & $C$ & $\tau$ \\
\hline 0.02 & $1 \mathrm{yr}$ & $7.62[3.47]$ & $1.03[1.03]$ & $-0.03[-0.02]$ \\
$(\mathrm{NH})$ & $5 \mathrm{yr}$ & $38.1[5.59]$ & $1.03[1.03]$ & $-0.03[-0.02]$ \\
\hline 0.04 & $1 \mathrm{yr}$ & $41.1[18.7]$ & $1.06[1.06]$ & $-0.05[-0.02]$ \\
$(\mathrm{NH})$ & $5 \mathrm{yr}$ & $205[29.7]$ & $1.06[1.05]$ & $-0.05[-0.00]$ \\
\hline 0.02 & $1 \mathrm{yr}$ & $4.50[1.84]$ & $1.00[1.00]$ & $0.04[0.04]$ \\
$(\mathrm{IH})$ & $5 \mathrm{yr}$ & $22.5[2.92]$ & $1.00[1.00]$ & $0.04[0.04]$ \\
\hline 0.04 & $1 \mathrm{yr}$ & $14.8[5.49]$ & $1.01[1.01]$ & $0.09[0.10]$ \\
$(\mathrm{IH})$ & $5 \mathrm{yr}$ & $73.8[8.47]$ & $1.01[1.00]$ & $0.09[0.10]$ \\
\hline \hline
\end{tabular}

$N_{i, j}(C=1, \tau=0 ; \alpha=0)$, we calculate $\chi^{2}$ value as

$$
\chi^{2}\left(C, \tau, \sin ^{2} \alpha\right)=\sum_{i, j} \frac{\left[N_{i, j}^{\text {null }}-N_{i, j}^{\bmod }\left(C, \tau, \sin ^{2} \alpha\right)\right]^{2}}{N_{i, j}^{\text {null }}} .
$$

Then $\chi^{2}$ is minimized by varying $C$ and $\tau$. The results, $\chi_{\min }^{2}$ and the corresponding values of $C$ and $\tau$ are listed in the Table I for $1 \mathrm{yr}$ and $5 \mathrm{yr}$ of exposure and for two different values of $\sin ^{2} \alpha$. According to Fig. 4, already after 1 year of data taking the statistical error in each bin is expected to be $\sim(2-4) \%$. However, as pointed out in Ref. 7], systematic errors for IceCube are not well defined yet and will likely dominate statistical errors. To illustrate this effect on the sensitivity to $\nu_{s}$ we performed $\chi^{2}$ analysis by introducing a $5 \%$ uncorrelated systematic error for each bin in addition to the statistical and correlated systematic (slope and normalization) errors. The values of $\chi^{2}$ and $(C, \tau)$ in this case are reported in Table I within brackets. The $\chi^{2}$ values in the Table should be compared with $\chi_{\min }^{2}=0$ which corresponds to zero mixing with $\nu_{s}$.

For $\mathrm{NH}$ the obtained $\chi^{2}$ values with 2 degrees of freedom mean that even with $5 \%$ additional systematic uncertainties, $\sin ^{2} \alpha=0.04$ or $\left|U_{\mu 4}\right|^{2}=0.02$ can be excluded at $>99.9 \%$ CL with one year of DeepCore data. As a result, the oscillation interpretation of LSND/MiniBooNE data 1, 2, that requires $\left|U_{\mu 4}\right|^{2} \gtrsim 0.03$ for $\Delta m_{43}^{2} \lesssim 1 \mathrm{eV}^{2}$ will be severely constrained.

In the case of $\sin ^{2} \alpha=0.02$ or $\left|U_{\mu 4}\right|^{2}=0.01$ five years of DeepCore data would be required to reach $\sim 95 \% \mathrm{CL}$ for exclusion. This is comparable with the current bound from the MINOS experiment 14. A mixing angle smaller than $\sin ^{2} \alpha \sim 0.02$ cannot be excluded with DeepCore data alone. For IH the effects is substantially smaller and only $\left|U_{\mu 4}\right|^{2} \gtrsim 0.02$ can be tested.

We estimate that the present uncertainty in $\Delta m_{32}^{2}$ reduces the sensitivity to $\nu_{s}$-mixing by factor of 2 , i.e. $\left|U_{\mu 4}\right|^{2}=0.02$ (for $\mathrm{NH}$ ) can be excluded at $>99.9 \% \mathrm{CL}$ after 4 years of the data taking.

\section{DISCUSSION AND CONCLUSION}

A combined analysis of the IceCube high-energy data which cover the MSW resonance dip at $\mathrm{TeV}$ energies, together with DeepCore low energy data will improve greatly the sensitivity to $\nu_{s}$ mixing parameters. Furthermore, a distinction between the flavor- and mass-mixing schemes will be possible since DeepCore event distributions are affected in the case of mass-mixing only, as we have studied here, while the IceCube event distributions are affected in both mixing schemes [5].

Notice that search for sterile neutrinos with IceCube and DeepCore are complementary to that with MINOS and other accelerator experiments. Indeed, IceCube measures the mass squared difference and mixing at much higher energies and with large matter effect. In scenarios with energy and enviroment dependent neutrino masses, the mass and mixing parameters may turn out to be substantially different in this two setups.

We considered the oscillation effects for specific mixing scheme and specific values of parameters and presented an illustrative analysis of simulated data. General analysis would include the sub-leading effects of the non-zero 1-3 mixing, possible effects of the CP-violation associated to existence of sterile neutrino, consideration of different values of $\left|U_{\tau 4}\right|$, etc.

In conclusion, we have found that mixing of the $\mathrm{eV}$ scale mass $\nu_{s}$ indicated by LSND/MiniBooNE, substantially affects the oscillation probabilities in the range 15 - $120 \mathrm{GeV}$ accessible to IceCube DeepCore experiment. The effect is opposite in $\nu$ - and $\bar{\nu}$ - channels and can reach $\sim O(1)$ size at the probability level. The $\nu_{s}$ mixing produces a shift and modifies shape of the oscillation dip. The effect is different for the normal and inverted mass hierarchies of the active neutrinos. Therefore, in principle, the hierarchy can be identified, if the sterile neutrinos with relatively large mixing exist. The zenith angle distributions of the $\nu_{\mu}^{C C}$ events in different energy ranges are changed. However, due to summation of $\nu-$ and $\bar{\nu}-$ signals the effect of $\nu_{s}-$ mixing and oscillations on the number of events is much weaker. Uncertainty in $\Delta m_{32}^{2}$ reduces sensitivity of DeepCore to sterile neutrinos. Nevertheless we find that Deep Core can probe the $\nu_{s}$ mixing down to $U_{\mu 4}=0.01-0.02$ for wide range of $\Delta m_{43}^{2}$.

\section{Acknowledgments}

We are grateful to D. F. Cowen and J. Koskinen for correspondence on the design and performance of the IceCube DeepCore. Work of S.R. was funded by NASA, and performed at and while under contract with the U.S. Naval Research Laboratory. S.R. would also like to thank the Abdus Salam ICTP for hospitality while completing the final manuscript. 
[1] A. Aguilar et al. [LSND Collaboration], Phys. Rev. D64 (2001) 112007. hep-ex/0104049.

[2] A. A. Aguilar-Arevalo et al. [The MiniBooNE Collaboration], Phys. Rev. Lett. 105 (2010) 181801, arXiv:1007.1150 [hep-ex]].

[3] H. Nunokawa, O. L. G. Peres and R. Zukanovich Funchal, Phys. Lett. B 562 (2003) 279 arXiv:hep-ph/0302039.

[4] S. Choubey, JHEP 0712 (2007) 014 arXiv:0709.1937 [hep-ph]].

[5] S. Razzaque and A. Yu. Smirnov, JHEP 1107, 084 (2011) arXiv:1104.1390 [hep-ph]].

[6] V. Barger, Y. Gao and D. Marfatia, Phys. Rev. D 85, 011302 (2012) arXiv:1109.5748 [hep-ph]].

[7] F. Halzen, arXiv:1111.0918 [hep-ph].

[8] R. Abbasi et al. [IceCube Collaboration], arXiv:1109.6096 [astro-ph.IM]

[9] C. H. Ha [for the IceCube Collaboration], arXiv:1201.0801 [hep-ex].

[10] O. Mena, I. Mocioiu and S. Razzaque, Phys. Rev. D 78, 093003 (2008) arXiv:0803.3044 [hep-ph]].

[11] E. Fernandez-Martinez, G. Giordano, O. Mena and I. Mocioiu, Phys. Rev. D 82, 093011 (2010)
arXiv:1008.4783 [hep-ph]].

[12] R. Abbasi et al. [IceCube Collaboration], arXiv:1111.2731 [astro-ph.IM].

[13] D. Fargion, D. D'Armiento, P. Di Giacomo and P. Paggi, Nucl. Phys. B 212-213 (2011) 146 arXiv:1103.2642 [astro-ph.HE]].

[14] P. Adamson et al. [MINOS Collaboration], Phys. Rev. Lett. 107, 011802 (2011) arXiv:1104.3922 [hep-ex]].

[15] A. Sousa and o. b. o. Collaboration, arXiv:1110.3455 [hep-ex].

[16] C. Giunti and M. Laveder, Phys. Rev. D 84, 093006 (2011) arXiv:1109.4033 [hep-ph]].

[17] P. Adamson et al. [MINOS Collaboration], arXiv:1202.2772 [hep-ex].

[18] A. M. Dziewonski and D. L. Anderson, Phys. Earth Planet. Interiors 25 (1981) 297.

[19] M. Honda, T. Kajita, K. Kasahara and S. Midorikawa, Phys. Rev. D 52, 4985 (1995) arXiv:hep-ph/9503439.

[20] D. F. Cowen and J. Koskinen, private communication.

[21] R. Abbasi et al. [IceCube Collaboration], Phys. Rev. D 83, 012001 (2011) arXiv:1010.3980 [astro-ph.HE]]. 